\title{
Detection of autoantibodies against carbonic anhydrase I and II in the plasma of patients with gastric cancer
}

\author{
AHMET MENTESE ${ }^{1}$, EVREN FIDAN ${ }^{2}$, AHMET ALVER ${ }^{3}$, SELIM DEMIR ${ }^{4}$, SERAP OZER YAMAN 5 , \\ AYSEGUL SUMER ${ }^{6}$, SAMI FIDAN ${ }^{7}$, HALIL KAVGACI ${ }^{8}$, IBRAHIM TURAN ${ }^{9}$ \\ ${ }^{1}$ Program of Medical Laboratory Techniques, Vocational School of Health Sciences, Karadeniz Technical University, Trabzon, Turkey \\ ${ }^{2}$ Department of Internal Medicine and Medical Oncology, Kanuni Teaching and Research Hospital, Trabzon, Turkey \\ ${ }^{3}$ Department of Medical Biochemistry, Faculty of Medicine, Recep Tayyip Erdogan University, Rize, Turkey \\ ${ }^{4}$ Department of Nutrition and Dietetics, Faculty of Health Sciences, Karadeniz Technical University, Trabzon, Turkey \\ ${ }^{5}$ Department of Medical Biochemistry, Faculty of Medicine, Karadeniz Technical University, Trabzon, Turkey \\ ${ }^{6}$ Department of Nursing, School of Health Services, Recep Tayyip Erdogan University, Rize, Turkey \\ ${ }^{7}$ Department of Gastroenterology, Faculty of Medicine, Karadeniz Technical University, Trabzon, Turkey \\ ${ }^{8}$ Department of Internal Medicine and Medical Oncology, Faculty of Medicine, Karadeniz Technical University, Trabzon, Turkey \\ ${ }^{9}$ Department of Genetic and Bioengineering, Faculty of Engineering and Natural Sciences, Gumushane University, Gumushane, Turkey
}

\begin{abstract}
Cancer is the second leading cause of death and gastric cancer is the fourth most common cancer type worldwide. Investigation of autoantibodies in cancer patients has been a popular research area in recent years. The aim of the current study was to investigate carbonic anhydrase I and II (CA I and II) autoantibodies in the plasma of subjects with gastric cancer based on the information and considerations of autoimmune relation of gastric cancer. Anti-CA I and II antibody levels were investigated by ELISA in plasma samples of fifty two patients with gastric cancer and thirty five healthy peers. Anti-CA I and II antibody titers of the gastric cancer group were significantly higher compared with the control group ( $p=0.004, p=0.0001$, respectively). Plasma anti-CA I levels of the metastatic group were lower than the non-metastatic group and this difference was found statistically significant $(p<0.05)$, but there was no statistical difference between plasma anti-CA II levels of the groups. CA I and II autoantibody titers in patients with gastric cancer were found higher compared to healthy subjects and the results suggest that these autoantibodies may be involved in the pathogenesis of gastric cancer.
\end{abstract}

Key words: autoantibody, carbonic anhydrase, gastric cancer, Helicobacter pylori.

(Cent Eur J Immunol 2017; 42 (1): 73-77)

\section{Introduction}

Malignant diseases progress with stimulation of autoimmunity that is characterized by formation of antibodies against their own antigens, and autoantibodies are detected in the sera of patients with solid tumors [1,2]. These autoantibodies are evaluated as early biomarkers of some types of cancer [3-5]. Cancer is the second leading cause of death and gastric cancer (GC) is the fourth most common cancer type worldwide [6]. Estimated new events and deaths from this disease in the US in 2015 were 24,590 and 10,720, respectively [7]. Approximately, $80 \%$ of gastric adenocarcinomas are associated with Helicobacter pylori infection and occur secondary to associated gastritis [8]. Autoantibodies developed against antigens of $H$. pylori and self-antigens of the organism are detected in the sera of subjects with GC [9].
Carbonic anhydrase (CA) is a metalloenzyme, which catalyzes the reversible hydration of carbon dioxide to bicarbonate. CA functions in many physiological and pathological processes, such as transport of carbon dioxide, $\mathrm{pH}$ regulation, ion transport, formation of stomach acidity, bone resorption, calcification, and tumorigenesis. Thus far 16 isoenzymes that differ from each other in tissue distribution, cell localization, catalytic activity and resistance to inhibitors, are described [10,11]. Most of these isoenzymes are expressed in the gastrointestinal tract [12]. In recent years CA I and II autoantibodies have been demonstrated in some autoimmune diseases and carcinomas, but mechanisms underlying this immune response have not been explained yet $[13,14]$.

The aim of our study was to evaluate CA I and II autoantibodies in the subjects with gastric cancer by the ELISA

Correspondence: Ahmet Mentese,Program of Medical Laboratory Techniques Vocational School of Health Sciences, Karadeniz Technical University, 61080 Trabzon, Turkey, e-mail: amentese028@gmail.com

Submitted: 6.03.2016, Accepted: 6.06.2016. 
method and provide a novel perspective to autoimmune basis of gastric cancer.

\section{Material and methods}

\section{Study group}

Elucidated approval was obtained from all patients and controls. Approval for the study was given by the local ethics committee. Fifty two newly diagnosed patients with GC (24 metastatic, 28 non-metastatic) as the study group and thirty five healthy peers as the control group were admitted to this study. There were 30 men and 22 women with a median age of 58 (range: 45-70) years in the study group, while there were 16 men and 19 women with a median age of 56 (range: 40-72) years in the control group. Patients were selected from individuals who applied to the Medical Oncology Clinic and were referred from other practitioners. Patients who had renal, coronary and liver failure, chronic inflammatory diseases, anemia, received chemotherapy, oral contraceptives and anticoagulants were excluded from the study. Patients were staged according to the seventh edition of the American Joint Committee on Cancer Manual [15]. Staging was assessed according to this classification. Out of 52 patients with gastric adenocarcinoma, $13.5 \%$ (7 patients) had stage II disease, $40.4 \%$ (21 patients) had stage III disease and $46.2 \%$ (24 patients) had stage IV disease. All patients had the same characteristic cancer cell type as an adenocarcinoma.

Five milliliters blood sample for each individual was obtained in vacutainer tubes with $\mathrm{K}_{3}$ EDTA. Tubes were centrifuged at $1800 \mathrm{~g}$ for 10 minutes. Plasma samples were stored at $-80^{\circ} \mathrm{C}$ until measurements.

\section{Determination of plasma autoantibody to CA I and II}

Plasma CA I and II autoantibodies were determined by ELISA according to the previously described method

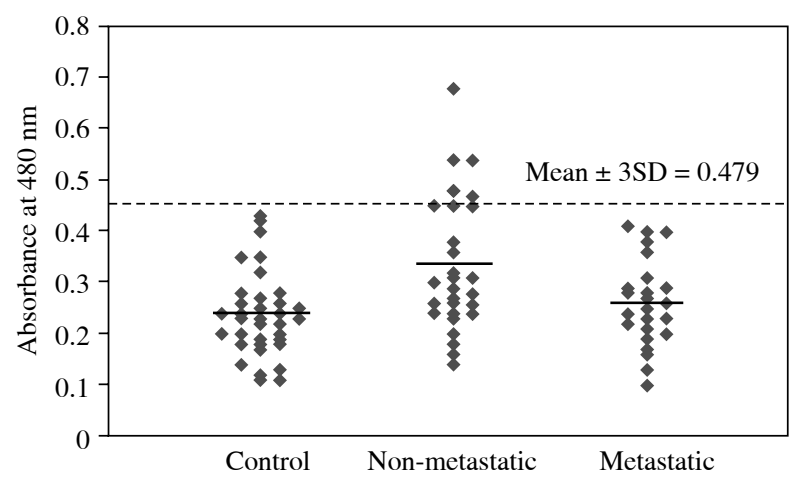

Fig. 1. Anti-CA I antibodies in plasma from patients with GC and healthy controls. The dotted line indicates the mean value $+3 \mathrm{SD}$ of healthy control plasma $\left(\mathrm{A}_{480}=0.479\right)$
[16]. Each sample was assayed in duplicate and the specific binding of plasma antibody to CA I or CA II was calculated as follows:

$$
\text { Specific binding }=\mathrm{OD}_{\text {coated }}-\mathrm{OD}_{\text {control }} \text {. }
$$

\section{Statistical analysis}

Statistical analysis was applied using a statistical package for the social sciences (Version 13.0, NY, USA) and MedCalc (Version 12.3, Mariakerke, Belgium) statistical software. Suitability for normal distribution was determined by Kolmogorov-Smirnov test. The differences between all groups were analyzed using Student's $t$-test for data with normal distribution. The receiver operating characteristic (ROC) curves were used to detect the discriminatory dominance of CA I and II autoantibodies for identification of GC. Sensitivity, specificity, negative predictive values (NPV) and positive predictive values (PPV) were determined with regard to ROC graphs for autoantibodies of CA I and II. $p<0.05$ was regarded as significant.

\section{Results}

Fifty two GC patients and thirty five healthy subjects were included in our study and 24 of cases were metastatic and the rest were non-metastatic subjects. There was no significant difference in terms of the median age between study and control groups. Levels of CA I and II autoantibodies in patients with GC and control subjects are shown in Figs. 1 and 2, respectively.

The mean absorbance value of CA I autoantibody for the healthy subjects was $0.236 \pm 0.082$ and the absorbance was higher than 0.479 , the mean absorbance $+3 \mathrm{SD}$ of healthy individuals, were identified as positive. Positive results were obtained in 5 (all of non-metastatic) out of 52 subjects with GC (Fig. 1). The mean absorbance value of the GC group $(0.299 \pm 0.117)$ was detected to be markedly

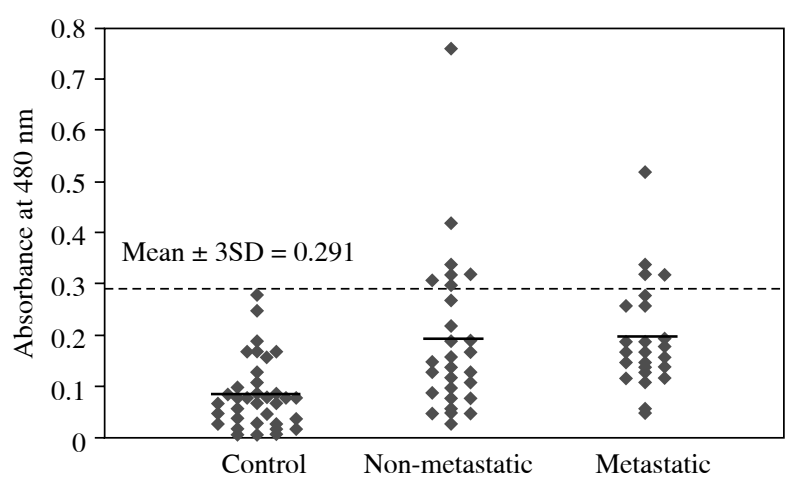

Fig. 2. Anti-CA II antibodies in plasma from patients with GC and healthy controls. The dotted line indicates the mean value $+3 \mathrm{SD}$ of healthy control plasma $\left(\mathrm{A}_{480}=0.291\right)$ 
Table 1. Anti-CA I and II antibody levels (ABSU)

\begin{tabular}{ccccc}
\hline & \multicolumn{4}{c}{ Groups } \\
\cline { 2 - 5 } & Patient & Control & Metastatic & Non-metastatic \\
\hline$n$ & 52 & 35 & 24 & 28 \\
\hline Anti-CA I & $0.299 \pm 0.117^{\mathrm{a}}$ & $0.236 \pm 0.082$ & $0.260 \pm 0.085^{\mathrm{c}}$ & $0.332 \pm 0.131$ \\
\hline Anti-CA II & $0.194 \pm 0.133^{\mathrm{b}}$ & $0.087 \pm 0.069$ & $0.196 \pm 0.120$ & $0.192 \pm 0.159$ \\
\hline Data are mean values $\pm S D ;{ }^{a} p=0.004$ compared with control, ${ }^{b} p=0.0001$ compared with control, ${ }^{c} p<0.05$ compared with non-metastatic
\end{tabular}

Table 2. ROC curve analysis of a CA I and II autoantibodies values and their sensitivity, specificity, PPV and NPV

\begin{tabular}{cccccc}
\hline & $\begin{array}{c}\text { Cut-off } \\
\text { Point }\end{array}$ & $\begin{array}{c}\text { Sensitivity (\%) } \\
(\mathbf{9 5 \%} \mathbf{C I})\end{array}$ & $\begin{array}{c}\text { Specificity (\%) } \\
(\mathbf{9 5 \%} \text { CI) }\end{array}$ & $\begin{array}{c}\text { PPV (\%) } \\
(\mathbf{9 5 \%} \text { CI) }\end{array}$ & $\begin{array}{c}\text { NPV (\%) } \\
(\mathbf{9 5 \%} \text { CI) }\end{array}$ \\
\hline \multirow{2}{*}{ Anti-CA I } & $>0.250$ & 61.5 & 68.6 & 74.4 & 54.5 \\
& & $(47.0-74.7)$ & $(50.7-83.1)$ & $(58.8-86.5)$ & $(38.8-69.6)$ \\
\hline \multirow{2}{*}{ Anti-CA II } & $>0.100$ & 78.4 & 73.5 & 81.6 & 69.4 \\
& & $(64.7-88.7)$ & $(55.6-87.1)$ & $(67.8-91.3)$ & $(51.9-83.7)$ \\
\hline
\end{tabular}

ROC - receiver operator characteristic; $P P V-$ positive predictive value; $N P V-$ negative predictive value

Anti-CA I Antibody

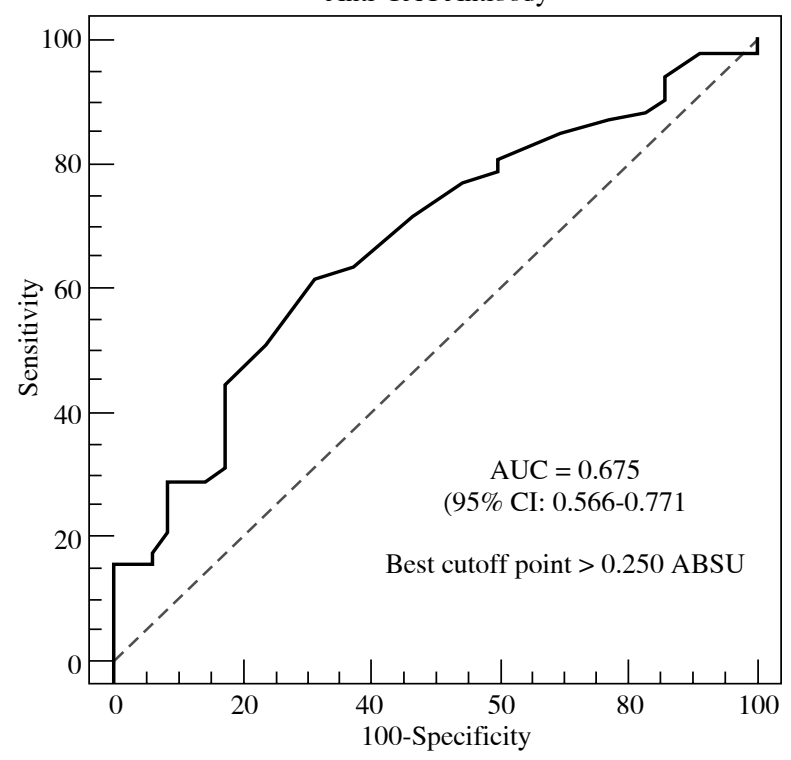

Fig. 3. ROC curve analysis of CA I autoantibody values in patients with $\mathrm{GC}$

higher $(p=0.004)$ compared with the healthy subjects (Table 1). The mean absorbance value of CA II autoantibody for the healthy subjects was $0.087 \pm 0.069$ and the absorbance was higher than 0.291 , the mean absorbance +3SD of healthy individuals, were identified as positive. Positive results were obtained in 11 (4 metastatic, 7 non-metastatic) out of 52 subjects with GC (Fig. 2). The mean absorbance value of the GC group $(0.194 \pm 0.133)$ was detected to be markedly higher $(p=0.0001)$ than of the healthy subjects (Table 1).

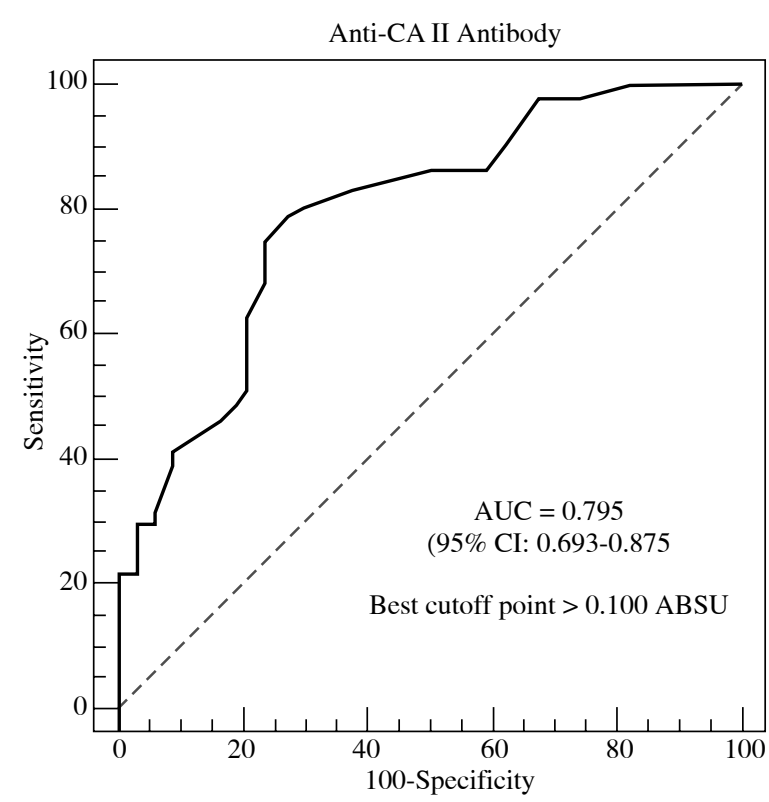

Fig. 4. ROC curve analysis of CA II autoantibody values in patients with $\mathrm{GC}$

Statistical analysis was performed by dividing the patients into two groups as metastatic and non-metastatic. Plasma anti-CA I levels of the metastatic group were lower than the non-metastatic group and this difference was found statistically significant $(p<0.05)$, but there was no statistically difference between plasma anti-CA II levels of the groups (Table 1).

Plasma CA I and II autoantibody levels were also evaluated using ROC curve analysis. Cut-off points, sensitivity, specificity, PPV and NPV for the parameters were demonstrated in Table 2 and Figs. 3 and 4. 


\section{Discussion}

Gastric cancer is the fourth most common cancer type and the second cause of cancer-related death. Late diagnosis is one of the reasons of its high mortality. In recent years, there have been therefore many research studies about identification of a new biochemical diagnostic marker for early detection of GC $[17,18]$. Autoantibodies in the blood sample of patients have been proposed as diagnostic biomarkers for early-stage diagnosis of cancers, as an increase in serum levels of certain autoantibodies has been shown to precede the development of disease symptoms and correlate with cancer incidence for many types of cancer [19]. Using serum antibodies as markers for cancer has some advantages. Primarily, cancer related autoantibodies exist in the bloodstream much earlier than serum antigens. Secondly, autoantibodies can have higher levels in the bloodstream compared to antigens [4].

The present study is the first report which shows an increased immune response to both CA I and II in GC patients. We found CA I and II autoantibody prevalence in patients with GC as $9.6 \%$ and $21.2 \%$, respectively (Figs. 1 and 2). In this study, an anti-CA I antibody cut-off level of 0.250 ABSU was related to NPV of $54.5 \%$ and PPV of $74.4 \%$, with $61.5 \%$ sensitivity and $68.6 \%$ specificity and an anti-CA II antibody cut-off level of 0.100 ABSU was related to NPV of $69.4 \%$ and PPV of $81.6 \%$, with $78.4 \%$ sensitivity and $73.5 \%$ specificity according to ROC curves. To date, there has been no gastric cancer specific biomarker, although autoimmunity against many autoantigens, such as p53, NY-ESO-1, mucin-1 (MUC1), c-myc, survivin, koc, p62, astrocyte elevated gene-1 protein (AEG1), matrix metalloproteinase-7 (MMP-7), 70 kilodalton heat shock proteins (Hsp70), carcinoembryonic antigen (CEA) and histone H2B are reported in GC. The sensitivity and specificity of these autoantibody markers range from $0 \%$ to $75 \%$ and from $71.7 \%$ to $100 \%$, respectively [18]. When viewed from this aspect, sensitivity and specificity of CA I and II autoantibodies were similar with previous autoantibody research in GC.

Carbonic anhydrase isoenzymes are virtually ubiquitous in living systems, have various functions in most normal mammalian and bacterial cells. CA I and II are the most widely distributed members of the CA family, being present almost in all tissues [20, 21]. Also, CA I and II have been demonstrated to be associated with gastrointestinal neoplasms and CA II has been mentioned as a new biomarker for gastrointestinal stromal tumors [22]. Increased autoimmunity against these isoenzymes has been reported in cancer and autoimmune diseases, but no mechanisms have been identified [13]. It has also been suggested that genetic, hormonal, and environmental influences may play a part in triggering autoimmunity [19]. The higher levels of autoantibodies in cancer patients can be explained by overexpression, aberrant expression, mutation, changes in protein half-lives, misfolding, aberrant degradation or abnormal posttranslational modification of the proteins $[18$, 23]. The increase in these autoantibodies may be a secondary epiphenomenon to proliferation of cancer cells. Iuchi et al. demonstrated that SOD-knock-out mouse developed CA II autoantibodies as a result of increased oxidative stress [24]. 4-hydroxy-2-nonenal (HNE), one of the end products of lipid peroxidation, modifies proteins and alters antigenic properties of them. Uchida et al. reported that CA II is a target for HNE in their study related with erythrocytes [25]. Increased CA autoantibody titers in the plasma of GC patients may be a result of increased oxidative damage in cancer tissue. Moreover, antibodies against to H.pylori antigens are determined in most of subjects with GC [26]. Helicobacter pylori is a gram negative, microaerophilic bacteria. This bacteria is known as the etiological agent of chronic active gastritis and as the factor responsible for most of the peptic ulcer cases and a co-factor for GC and MALT-lymphoma [27, 28]. Helicobacter pylori has $\alpha$ - and $\beta$-carbonic anhydrase enzymes. These enzymes provide acid acclimation of the pathogen in the gastric tract. Helicobacter pylori $\alpha$-class CA (hp $\alpha \mathrm{CA})$ has been sequenced from individuals with varied gastric mucosal lesions, such as gastritis, ulcer and GC [10]. An in vitro study demonstrated that antibodies against CA of this bacteria might have formed an autoimmune response to human CA as a result of molecular mimicry [29, 30]. In a study from our laboratory, anti-CA II titers of $H$. pylori positive subjects were found higher than $H$. pylori negative subjects and control groups (unpublished data). Most of the CA isoenzymes including CA I and II are found in the gastrointestinal system cells. CA IX and XII are related to carcinogenesis and have an increased expression in malignant tumor cells [12]. Immune response to CA I and II in GC subjects may be a cross-reactivity resulting from other CA isozymes that mimic these ones.

There are many methods, such as ELISA, western blot, protein microarrays, agglutination assays, immunoblotting, flow cytometry, immunoprecipitation and immunofluorescence for determination of autoantibodies in the sera of cancer patients. ELISA is frequently used for detection of autoantibodies in blood samples due to its cheapness, easiness and quickness as compared to the other assay [18, 31]. Besides, it has been mostly used for evaluation of CA I and II autoantibodies in different pathological conditions in previous reports $[11,14,16,32]$. We have therefore preferred to determine autoantibody levels using ELISA in this study.

Plasma anti-CA I levels of the non-metastatic group were higher than the metastatic group and this difference was statistically significant. The finding that in non-metastatic patients elevated the immune response to CA I may have prevented tendency to metastasis. This may be related to the ability of cancer cells to escape from the immune system. For this reason antibody formation in the meta- 
static subjects may be less than in the non-metastatic ones. The major limitation of the current study may be a relatively small number of patients. Further larger-scale prospective and molecular studies are required for a fully understood autoimmunity mechanism of CA I and II in GC.

In the present study, CA I and II autoantibodies are detected in GC subjects, but the pathogenic role of these antibodies remain uncertain. It shows the need for further trials to evaluate the significance of CA autoantibody production in GC subjects.

\section{Ethical Approval}

All procedures performed in studies involving human participants were in accordance with the ethical standards of the institutional and/or national research committee and with the 1964 Helsinki Declaration and its later amendments or comparable ethical standards.

The authors declare no conflict of interest.

\section{References}

1. Abu-Shakra M, Buskila D, Ehrenfeld M, et al. (2001): Cancer and autoimmunity: Autoimmune and rheumatic features in patients with malignancies. Ann Rheum Dis 60: 433-441.

2. Ortona E, Pierdominici M, Berstein L (2014): Autoantibodies to estrogen receptors and their involvement in autoimmune diseases and cancer. J Steroid Biochem Mol Biol 144: 260267.

3. Chapman CJ, Murray A, McElveen JE, et al. (2008): Autoantibodies in lung cancer: Possibilities for early detection and subsequent cure. Thorax 63: 228-233.

4. Zhong L, Ge K, Zu JC, et al. (2008): Autoantibodies as potential biomarkers for breast cancer. Breast Cancer Res 10: R40.

5. Lowe FJ, Shen W, Zu J, et al. (2014): A novel autoantibody test for the detection of pre-neoplastic lung lesions. Mol Cancer 13: 78 .

6. Wei ZW, Xia GK, Wu Y, et al. (2015): CXCL1 promotes tumor growth through VEGF pathway activation and is associated with inferior survival in gastric cancer. Cancer Lett 359: 335-343.

7. Siegel RL, Miller KD, Jemal A (2015): Cancer statistics, 2015. CA Cancer J Clin 65: 5-29.

8. Sipponen P (2002): Gastric cancer: pathogenesis, risks, and prevention. J Gastroenterol 37: 39-44.

9. Heneghan MA, McCarthy CF, Janulaityte D, et al. (2001): Relationship of anti-Lewis $\mathrm{x}$ and anti-Lewis $\mathrm{y}$ antibodies in serum samples from gastric cancer and chronic gastritis patients to Helicobacter pylori-mediated autoimmunity. Infect Immun 69: 4774-4781.

10. Supuran CT (2008): Carbonic anhydrases: Novel therapeutic applications for inhibitors and activators. Nat Rev Drug Discov 7: 168-181.

11. Mentese A, Guven S, Sumer A, et al. (2013): Serum anti-carbonic anhydrase I and II antibodies and polycystic ovary syndrome. Turk J Biochem 38: 43-48.
12. Kivela AJ, Kivela J, Saarnio J, et al. (2005): Carbonic anhydrases in normal gastrointestinal tract and gastrointestinal tumours. World J Gastroenterol 11: 155-163.

13. Adamus G (2009): Autoantibody targets and their cancer relationship in the pathogenicity of paraneoplastic retinopathy. Autoimmun Rev 8: 410-414.

14. Alver A, Senturk A, Cakirbay H, et al. (2011): Carbonic anhydrase II autoantibody and oxidative stress in rheumatoid arthritis. Clin Biochem 44: 1385-1389.

15. Edge SB, Byrd DR, Compton CC, et al. editors. AJCC cancer staging manual (7th ed). Springer, New York 2010.

16. Kino-Ohsaki J, Nishimori I, Morita M, et al. (1996): Serum antibodies to carbonic anhydrase I and II in patients with idiopathic chronic pancreatitis and Sjögren's syndrome. Gastroenterology 110: 1579-1586.

17. Fedele C, Carvalho S, Riccio G, et al. (2014): Effects of a human compact anti-ErbB2 antibody on gastric cancer. Gastric Cancer 17: 107-115.

18. Werner S, Chen H, Tao S, et al. (2015): Systematic review: Serum autoantibodies in the early detection of gastric cancer. Int J Cancer 136: 2243-2252.

19. Zaenker P, Ziman MR (2013): Serologic autoantibodies as diagnostic cancer biomarkers-A review. Cancer Epidemiol Biomarkers Prev 22: 2161-2181.

20. Sly WS, Hu PY (1995): Human carbonic anhydrases and carbonic anhydrase deficiencies. Annu Rev Biochem 64: 375401 .

21. Boztas M, Cetinkaya Y, Topal M, et al. (2015): Synthesis and carbonic anhydrase isoenzymes I, II, IX, and XII inhibitory effects of dimethoxybromophenol derivatives incorporating cyclopropane moieties. J Med Chem 58: 640-650.

22. Parkkila S, Lasota J, Fletcher JA, et al. (2010): Carbonic anhydrase II. A novel biomarker for gastrointestinal stromal tumors. Mod Pathol 23: 743-750.

23. Pedersen JW, Wandall HH (2011): Autoantibodies as biomarkers in cancer. Lab Medicine 42: 623-628.

24. Iuchi Y, Okada F, Onuma K, et al. (2007): Elevated oxidative stress in erythrocytes due to SOD1 deficiency causes anemia and triggers autoantibody production. Biochem J 402: 219227.

25. Uchida K, Hasui Y, Osawa T (1997): Covalent attachment of 4-hydroxy-2-nonenal to erythrocyte proteins. J Biochem 122: 1246-1251.

26. Bergman MP, D'Elios MM (2010): Cytotoxic T cells in H. pylori-related gastric autoimmunity and gastric lymphoma. J Biomed Biotechnol; Article ID: 104918, DOI: 10.1155/2010/104918.

27. Buckley MJ, O’Morain CA (1998): Helicobacter biology-discovery. Br Med Bull 54: 7-16.

28. Zhang S, Lee DS, Morrissey R, et al. (2014): Early or late antibiotic intervention prevents Helicobacter pylori-induced gastric cancer in a mouse model. Cancer Lett 355: 106-112.

29. Kountouras J, Zavos C, Chatzopoulos D (2005): Autoimmune pancreatitis, Helicobacter pylori infection, and apoptosis: a proposed relationship. Pancreas 30: 192-193.

30. Bhatia M (2005): Molecular mimicry in autoimmune pancreatitis: An interesting idea. J Cell Mol Med 9: 745.

31. Woodard KM, Chapman CJ (2008): Lung cancer-can autoantibodies provide an aid to diagnosis? Expert Opin Med Diagn 2: 911-923.

32. Alver A, Mentese A, Mentese U, et al. (2014): Anti-carbonic anhydrase II antibodies in end-stage renal disease patients. Med Princ Pract 23: 331-335. 